\title{
Interaction of nitrophenols with lipids at the air/water interface
}

\author{
Z. $\operatorname{Kozarac}^{1}$ ), A. Dhathathreyan²), and D. Möbius²) \\ $\left.{ }^{1}\right)$ Rudjer Boskovic Institute, Center for Marine Research, Zagreb, Croatia, Yugoslavia \\ 2) Max-Planck Institut für Biophysikalische Chemie, Göttingen, F.R.G.
}

\begin{abstract}
The uptake of ortho and para nitrophenol to charged and neutral lipid monolayers spread at the air/solution interface was studied by reflection spectroscopy. The adsorption characteristics of the two nitrophenols have been studied by measuring the surface pressure and surface potential as a function of molecular area of the different lipid monolayers in the presence of nitrophenols in the subphase. The results have been interpreted in terms of the electrostatic interaction between the negatively charged dissociated phenolate ions and the positively charged head group of dioctadecyldimethylammonium bromide monolayers.
\end{abstract}

Key words: Nitrophenol, dioctadecyldimethylammonium bromide, phospholipid monofilm, electrostatic interaction.

\section{Introduction}

Nitroaromatic compounds are frequently used as pesticides, explosives, and dyes. They are also used in chemical industry as solvents and precursors for the production of aminoaromatic derivatives. Consequently, they appear in waste waters, as well as in all natural aquatic systems, thereby representing highly dangerous pollutants of the environment [1]. Nitrophenols are listed as priority pollutants by the Environmental Protection Agency [2]. The study of their interaction with natural and/or artificial constituents of natural and polluted aquatic systems is of great importance and may increase the understanding of their possible role in the environment. Complex monolayers [3] that are composed of different artificial and or natural lipid components can be studied at the air/water interface by monolayer techniques which provide methods to organize appropriate molecules in a planned way and to study interactions at the interface under controlled conditions. Information on the molecular organization of such monolyers can be obtained from spectroscopic measurements, especially with the recently developed reflection spectroscopy $[4,5]$. Here we report monolayer studies of adsorption of ortho and para-nitrophenols to various lipid compounds at air/solution interface using reflection spectroscopy. The action of the dissociated phenolate ions on the monolayer components has been studied by measuring the surface pressure and surface potential of the lipid films as a function of their molecular area $(\Pi-A$ and $\Delta V-A)$ on substrates with and without the nitrophenol molecules.

\section{Materials and methods}

Ortho-nitrophenol (ONP), para-nitrophenol (PNP), p.a.grade and dioctadecyldimethylammonium bromide (DOMA) were purchased from Sigma Chemie. Trimethyloctadecylammonium bromide (TOMA) was purchased from Merck. Eicosyltrimethylammonium bromide (ETAB) was obtained from $\mathrm{E}$. Schuchardt and used without further purification. Dipalmitoylphosphatidyl choline (DPPC) was purchased from Larodan Chemicals. The substrates used for $\mathrm{pH}=3.0 \pm 0.1,5.2 \pm 0.1$, and $10.1 \pm 0.1$ were composed of $\mathrm{HCl}$ (Baker analyzed reagent), and tris-hydroxyamino methane (Merck) with or without nitrophenols (the ionic strengths used were of the order or $2 \mathrm{mM}$ ). To the buffer solution for $\mathrm{pH}=8.3$ $\pm 0.1,0.55 \mathrm{M} \mathrm{NaCl}$ (Merck) and $3 \times 10^{-2} \mathrm{M} \mathrm{NaHCO}_{3}$ (Merck) were added in order to simulate the conditions of real aquatic systems. Deionized water from a Milli-Q system (Millipore) was used for preparing the subphase. The $\mathrm{pH}$ of the subphase was controlled before and after each measurement. Chloroform was used as the spreading solvent for the lipid monolayers. The commercial product $\left(\mathrm{CHCl}_{3}, \mathrm{Baker}\right.$ Chemicals, p.a. grade) was run through a $1-\mathrm{m}$ - 

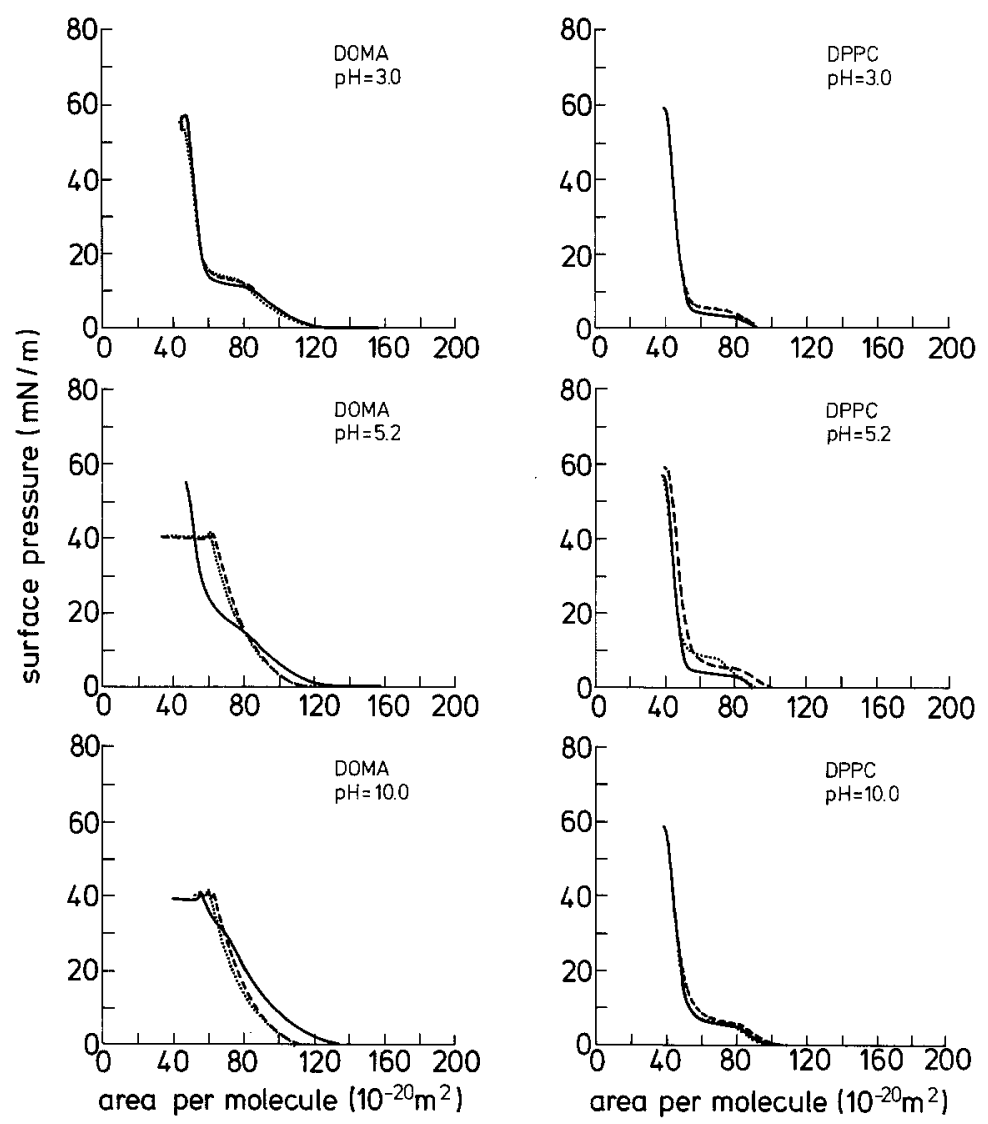

Fig. 1. Surface pressure-molecular area $(\Pi-A)$ isotherms of a) DOMA and b) DPPC monolayers on pure subphase (solid lines), on subphase with ortho-nitrophenol $2 \times 10^{-4} \mathrm{M}$ (dashed line) and on para-nitrophenol, $2 \times 10^{-4} \mathrm{M}$ (dotted line) at different $\mathrm{pH}$ values $\mathrm{i}$ ) 3.0 \pm 0.1 ; ii) $5.2 \pm 0.1$; and iii) $10.1 \pm 0.1$

column filled with alumina (Woelm, B-Super I) and used after addition of 1 vol $\%$ ethanol.

Monolayers were prepared at the air/solution interface in a circular multicompartment trough of the type designed by Fromherz [6]. The surface pressure was measured with a 1-cm-wide filter paper Wilhelmy balance [3] with an accuracy of $0.1 \mathrm{mN} / \mathrm{m}$. The $\Pi-A$ and $\Delta V-A$ curves were obtained $5 \mathrm{~min}$ after the monolayer was spread and the rate of compression was about $5 \AA^{2} / \mathrm{mol} / \mathrm{min}$. Reflection spectroscopic measurements were performed on monolayers spread on the aqueous substrate and then compressed to the required surface pressure (also called initial surface pressure $\Pi_{i}$ ). The reflection spectrometer for measurement under normal incidence of light and a modified instrument for the measurement of reflection spectra under oblique incidence of linearly polarized light [5] were used. The reflection was measured and expressed as the difference $\Delta R$ in the reflectivity of the solution surface covered with a monolayer to that of the clean water surface.

\section{Results and discussions}

\section{a) Surface pressure - area isotherms}

It is well known that adsorption of dissolved substances from a bulk solution to the surface alters both surface tension and surface potential at the air/solution interface. In the case of nitrophenol it can be expected that a large fraction of the undissociated molecules [7] would accumulate at the air/solution interface. Our measurements of surface pressure change $\Delta \pi$, of both ONP and PNP solutions at a relatively high concentration of $2 \times 10^{-4} \mathrm{M}$ did not show any appreciable change in surface pressure with time for any $\mathrm{pH}$ and salt concentration used. This is in agreement with earlier results [8]. PNP in aqueous solution almost did not influence the water surface tension and even in very concentrated solutions of about $10^{-1} \mathrm{M}$, a decrease of about only $6 \mathrm{mN} / \mathrm{m}$ in comparison to the surface tension of water was obtained. This indicates that both PNP and ONP are not very surface active.

If lipid monolayers are present on top of the solution both electrostatic and hydrophobic interactions could take place between the lipid molecules and the solute molecules. The binding of the solute molecules to lipid monolayers results in a change in the monolayer characteristics. The interactions of the two nitrophenols, in the subphase at different $\mathrm{pH}$ values with 


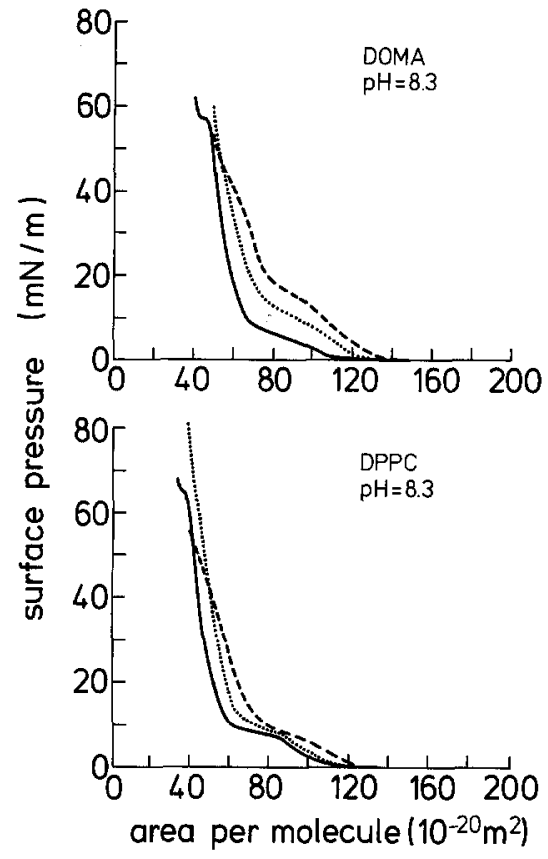

Fig. 2. Surface pressure-molecular area $(\Pi-A)$ isotherms of a) DOMA and b) DPPC monolayers on pure subphase (solid lines), on ortho-nitrophenol (dashed lines), and on para-nitrophenol (dotted lines), $2 \times 10^{-4} \mathrm{M}$ at $\mathrm{pH}=8.3 \pm 0.1$ (corresponding to natural aquatic systems)

the different lipid monolayers have been investigated. The $p K$ values of ONP and PNP are 7.22 and 7.15, respectively. The surface pressure-molecular area $(\Pi-A)$ isotherms of monolayers spread on solutions of ONP and PNP at different $\mathrm{pH}$ values of $3.0 \pm 0.1$; $5.2 \pm 0.1$ and $10.1 \pm 0.1$ are shown in Fig. 1 (DOMA and DPPC). Figure 2 shows the corresponding isotherms on the subphase of $\mathrm{pH}=8.3 \pm 0.1$ simulating the natural aquatic systems. It is seen from these figures that for all $\mathrm{pH}$ values the isotherms of DPPC monolayers do not show any appreciable change upon addition of ONP and PNP to the subphase.

In the case of DOMA monolayers on subphase without the nitrophenols it is seen that the transition occuring in the $\Pi-A$ curve at $\mathrm{pH}=3.0$ around 10 $\mathrm{mN} / \mathrm{m}$ shifts to larger surface pressures at $\mathrm{pH}=5.2$ and 10.0 , respectively. It is known that the properties of DOMA monolayers depend strongly on the different counterions in the subphase and the lateral interactions in the DOMA monolayer show a pronounced counterion specificity [9]. At $\mathrm{pH}=3.0$ the counterion in the subphase is chloride, giving rise to a sharp phase transition from the liquid condensed to the solid phase. As the $\mathrm{pH}$ of the subphase is changed from 3.0 to 5.2 and then to 10.0 it is seen that the phase transition in the $\Pi-A$ curve for DOMA monolayer becomes less pronounced and the pressure at which this occurs shifts to higher values. This change of the DOMA monolayer to a nearly expanded film is due to hydroxyl ions competing with chlorides at $\mathrm{pH}$ values larger than 3.0. This phenomenon has been related to the hydration of the counterions with the chloride ion being less hydrated than the hydroxyl ion [9].

In the case of both ONP and PNP in the subphase, at $\mathrm{pH}=3.0$ the fraction of phenolate ions is small. As the $\mathrm{pH}$ of the subphase is changed from 3.0 to first 5.2 and then to 10.0, an increasing fraction of the nitrophenol dissociates and the phenolate ions compete with the chloride and hydroxyl ions. The incorporation of the nitrophenolate which is less hydrated than chloride or hydroxyl anions leads to a condensation at small surface pressures. At higher surface pressures of more than $30 \mathrm{mN} / \mathrm{m}$, the area per DOMA molecule is slightly increased in comparison to the isotherms in the absence of ONP or PNP. We attribute this effect to a possible incorporation of the phenolate into the DOMA monolayer differing from the binding of the inorganic anions which may be located below the positively charged head groups. The DOMA monolayers at $\mathrm{pH}=8.3$ with $0.55 \mathrm{M} \mathrm{NaCl}$ in the subphase show the phase transition occuring between 2 and $10 \mathrm{mN} /$ $\mathrm{m}$. Here again the chloride and nitrophenolate anions compete with each other. The nitrophenolate causes an expansion of the DOMA monolayer.

It is known that almost all monolayers show an expansion effect with high concentration of $\mathrm{NaCl}$ in the subphase [10]. The decrease in the value of the collapse pressure for DOMA monolayers on ONP and PNP at $\mathrm{pH}$ values of 5.2 and 10.0 may be attributed to the liquid expanded state of the monolayers which have lower collapse pressure as compared to monolayers in the solid state [11].

The preferential interaction of both ONP and PNP to DOMA monolayer, as seen from the $\Pi-A$ curves in comparison to that of DPPC monolayers, indicates that the ionic interactions of the positively charged DOMA with nitrophenolate anions are stronger than hydrogen bonding and hydrophobic interactions. If such interactions were contributing appreciably to binding it would be apparent at $\mathrm{pH}=3.0$ where practically no nitrophenolate is present. Furthermore, these interactions would also be effective in the binding of ONP and PNP to DPPC. 
Table 1. Surface potential $\Delta V$ for DPPC and DOMA monolayers on subphase with and without ONP and PNP for different $\mathrm{pH}$ values $(\Pi=30 \mathrm{mN} / \mathrm{m})$

\begin{tabular}{llcccc}
\hline \multicolumn{5}{c}{$\begin{array}{l}\Delta V / m V \\
\text { pH of the subphase }\end{array}$} \\
\hline Monolayer & & 3.0 & 5.2 & 10.0 & $\left.8.3^{\mathrm{a}}\right)$ \\
DPPC & pure subphase & 640 & 590 & 540 & 840 \\
& ONP & 640 & 555 & 550 & - \\
& PNP & 640 & 595 & 560 & 800 \\
& & & & & \\
DOMA & pure subphase & 925 & 990 & 695 & 830 \\
& ONP & 875 & 650 & 520 & - \\
& PNP & 865 & 450 & 325 & 640 \\
\hline
\end{tabular}

a) with $0.55 \mathrm{M} \mathrm{NaCl}$ and $3 \times 10^{-2} \mathrm{M} \mathrm{NaHCO}_{3}$

The binding of both ONP and PNP from the bulk solution at $\mathrm{pH}=5.2$ (lower than the $\mathrm{pH}$ values of $\mathrm{ONP}$ and PNP) to DOMA monolayers here can not be taken as evidence against the ionic nature of the interaction. The removal of the nitrophenolate ions from the bulk upon association with the positively charged molecules of the DOMA monolayer shifts the equilibrium and induces further ionization of the nitrophenol molecules in the bulk. The local $\mathrm{pH}$ under the present experimental conditions (bulk pH=5.2) at about $10 \AA$ below the surface of the monolayer was estimated to be 6.2 from the Gouy-Chapman theory [12].

\section{b) Surface potentials}

The formation of a monolayer causes a change in the surface potential with respect to the monolayer free surface (measured as $\Delta V=V_{\text {mono }}-V_{\mathrm{H}_{2} \mathrm{O}}$ ). The presence of solute molecules in the subphase interacting with the monolayers causes an additional change of the surface potential value. In Table 1 the values of surface potential $\Delta V$ for close-packed monolayers of DOMA and DPPC are given for subphase with and without nitrophenols. Only relative changes of $\Delta V$ values indicating the influence of the substrate upon film characteristics should be considered. No appreciable change of the surface potential values can be seen for DPPC monolayers in agreement with the lack of binding of nitrophenol molecules. In the case of DOMA monolayer the surface potential values with ONP and PNP $(\mathrm{pH}=5.2)$ decrease considerably in comparison to the subphase without nitrophenol. It is also to be noted that the $\Delta V$ value for DOMA in the presence of PNP at alkaline $\mathrm{pH}$ shows the lowest value of $325 \mathrm{mV}$ on any subphase.

It must be pointed out that the measured values of surface potential for $\mathrm{pH}=8.3$ (Table 1) cannot be directly compared to those at other $\mathrm{pH}$ values because of different ionic strengths. The surface potential data support the conclusions drawn from the $\Pi-A$ curves for both DOMA and DPPC monolayers.

\section{c) Light reflection spectroscopy}

The interaction of the nitrophenol from the bulk solution with two different lipid monolayers has been studied by reflection spectroscopic technique. The method [4] is based on the modification of light reflection from the air/water interface due to the presence of chromophores at the interface. This technique has been used to study the adsorption of dyes [13-15], and lipid-protein interactions at the air/solution interface [16-18]. It provides independent information on the density and orientation of the bound molecules.

Accumulation of the nitrophenol molecules at the solution/air interface in the presence of the different lipid monolayers results in the change in the reflectivity from the interface. This change depends on the chromophore density and their orientation at the interface. Figure 3 shows the reflection signal of the phenolate ions adsorbed to DPPC (curve 1), DOMA, TOMA, and ETAB (curves 2, 3, and 4), respectively. Here the monolayers were first spread and then compressed to the desired initial surface pressure by holding the films at constant area and hence a constant head group density. During adsorption of ONP or PNP at constant monolayer area, the surface pressure may change as can be seen from the $\Pi-A$ curves (Figs. 1 and 2). Spectroscopic measurements were made when equilibrium was reached. The reflection signal obtained with DPPC monolayer spread on PNP is similar to that obtained for pure PNP solution without any monolayer. Curves 2, 3, and 4 show the paranitrophenolate anions adsorbing to the positive charge of the quarternary ammonium salt. Since only chromophores present at the interface contribute to the enhanced light reflection without any contribution from the chromophores in the bulk solution, the reflection spectra confirm the uptake of the nitrophenolate ions by the positively charged head groups of the monolayers. TOMA and ETAB monolayers are not very stable on water and hence, all further experiments were carried out with DOMA monolayers. 


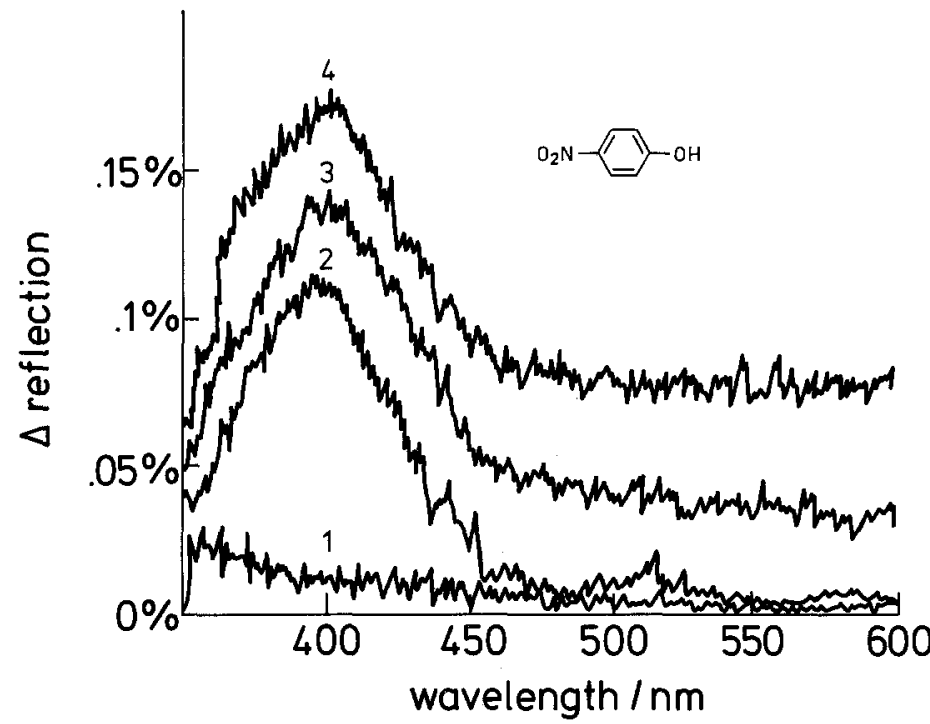

Fig. 3. Reflection spectra of para-nitrophenol adsorbed to i) DPPC; ii) TOMA; iii) ETAB, and iv) DOMA monolayers compressed to $30 \mathrm{mN} / \mathrm{m}, \mathrm{pH}=10.0 \pm 0.05$. Concentration of PNP inn subphase $=2 \times 10^{-4} \mathrm{M}$

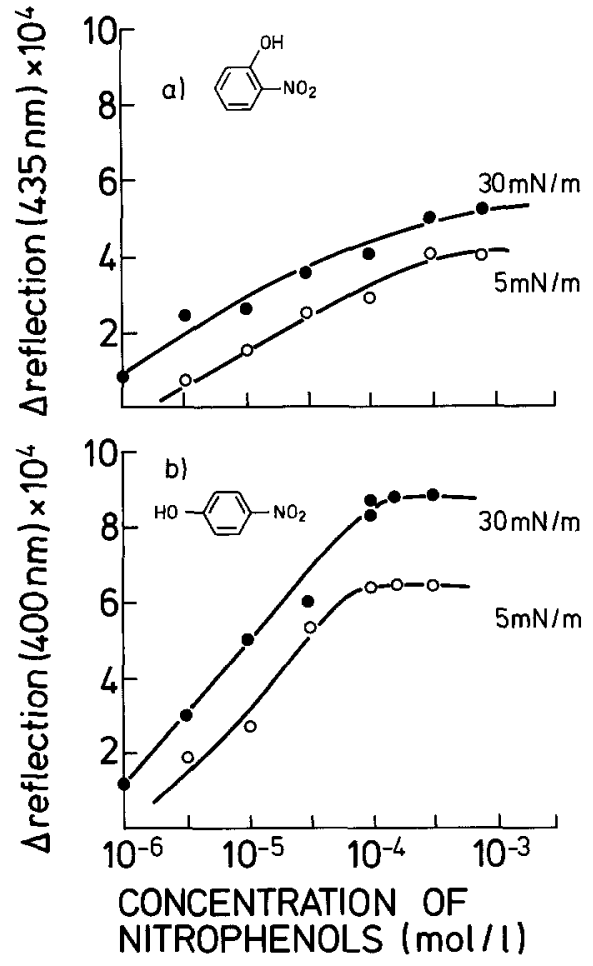

Fig. 4. The enhanced light reflection $\Delta R$ for a) ortho-nitrophenol $(\lambda$ $=435 \mathrm{~nm})$ and $\mathrm{b})$ para-nitrophenol $(\lambda=400 \mathrm{~nm})$ adsorbed to DOMA monolayers vs. concentration of nitrophenols at different initial surface pressures, i) $5 \mathrm{mN} / \mathrm{m}$; ii) $30 \mathrm{mN} / \mathrm{m}$
The dependence of $\Delta R$ values upon nitrophenol concentration in solution for two different initial surface pressures of DOMA monolayers is presented in Fig. 4. The interaction of both ONP and PNP can be followed by reflection spectroscopy in the concentration range between $5 \times 10^{-6}$ to $10^{-3} \mathrm{M}$. To ensure that equilibrium was reached in the uptake of the nitrophenol molecules by the monolayers within a reasonable time during the measurement of $\Pi-A$ isotherms we chose higher concentrations from the adsorption isotherms (Fig. 4).

The dominant role of electrostatic interactions seen here has also been found in previous studies of lipid protein interactions $[16,17]$. All these studies show a different kinetics for changes in $\Delta \Pi$ and $\Delta R$ with time depending on monolayer density. In the case of DOMA-nitrophenol interactions no significant dependence of changes in $\Delta \Pi$ and $\Delta R$ with time on the monolayer initial surface pressure was observed. The results shown in Fig. 4 and Table 2 show that the equi-

Table 2. Dependence of reflection $\Delta R(435 \mathrm{~nm})$ on the initial surface pressure of DOMA monolayers at the air/solution interface. Subphase: $5 \times 10^{-4} \mathrm{M}$ ONP in $2 \times 10^{-2} \mathrm{M}$ Tris buffer, $\mathrm{pH}=10.0 \pm 0.1$

\begin{tabular}{llll}
\hline $\begin{array}{l}\Pi \text { initial } \\
(\mathrm{mN} / \mathrm{m})\end{array}$ & $\begin{array}{l}A \\
\left(\mathrm{~nm}^{2}\right)\end{array}$ & $\begin{array}{l}\Delta R \\
(435 \mathrm{~nm})\left(10^{3}\right)\end{array}$ & $\begin{array}{l}\Delta R^{*} A \\
\left(\mathrm{~nm}^{-2}\right)\end{array}$ \\
\hline 5 & 0.965 & 3.125 & 3.01 \\
10 & 0.882 & 3.675 & 3.24 \\
15 & 0.816 & 3.825 & 3.12 \\
20 & 0.771 & 4.750 & 3.66 \\
30 & 0.706 & 4.825 & 3.40 \\
\hline
\end{tabular}




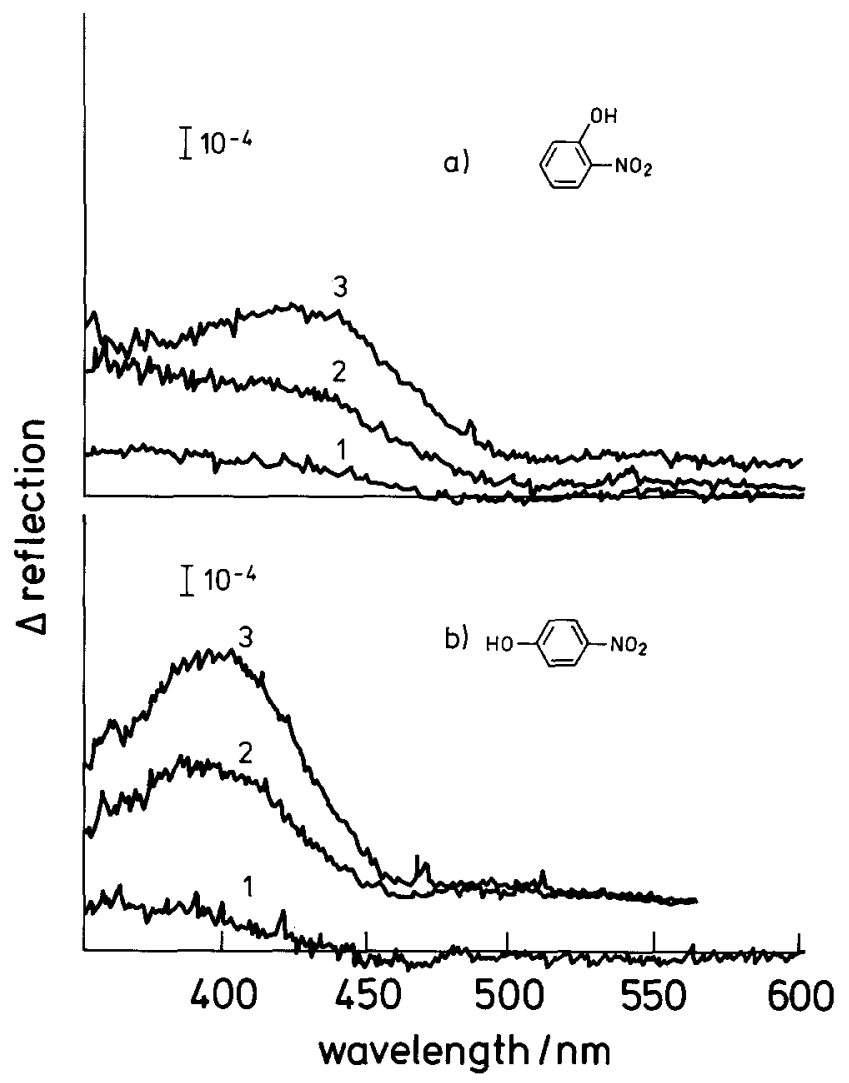

Fig. 5. Reflection spectra obtained for a) ortho-nitrophenol and b) para-nitrophenol $2 \times 10^{-4} \mathrm{M}$ in the absence (curve 1) and in the presence of DOMA monolayers (curve 2), $\mathrm{pH}=8.3$ (curve 3 ) and $\mathrm{pH}=10.0$, surface pressure $=30 \mathrm{mN} / \mathrm{m}$

librium values of $\Delta R$ increase slightly with increasing initial surface pressure. At the same time only small fluctuations in surface pressure values were obtained. The nitrophenol molecules seem to bind to the same extent at the monolayer, whether the monolayer is in the fluid or in the solid state (before interaction with nitrophenols). Highest reflection signal was obtained for nitrophenol in the subphase at alkaline $\mathrm{pH}$. This is to be expected because most of the nitrophenol molecules are in the dissociated state and the phenolate ions accumulate at the interface to interact with the positively charged DOMA. In Table 2 are given the $\Delta R$ values for different initial surface pressures for DOMA monolayer spread on ONP at $\mathrm{pH}=10.0$. It is seen that the product $\Delta R^{*} A$ where $A$ is the molecular area of the monolayer for the different surface pressures is increasing slightly in going from $\Pi=5 \mathrm{mN} / \mathrm{m}$ to $\Pi=$ $30 \mathrm{mN} / \mathrm{m}$. This indicates that the reflection signal is proportional to the surface density of the adsorbed

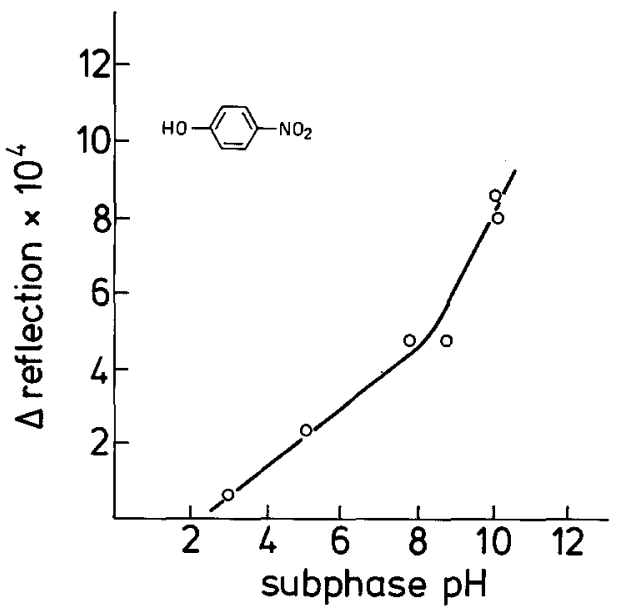

Fig. 6. Dependence of reflection $\Delta R(\lambda=400 \mathrm{~nm})$ on $\mathrm{pH}$ of the subphase for para-nitrophenol, $2 \times 10^{-4} \mathrm{M}$, adsorbed to DOMA at $\Pi=30 \mathrm{mN} / \mathrm{m}$

phenolate ions and the partition coefficient of ONP between bulk and the interface changes only slightly.

Reflection spectra of ONP and PNP at two different pH values of the subphase are presented in Fig. $5 \mathrm{a}$ ) and b). The dependence of $\Delta R$ at $\lambda_{\max }=400 \mathrm{~nm}$ for PNP adsorbing to DOMA monolayers at different $\mathrm{pH}$ values of the subphase is shown in Fig. 6. For all $\mathrm{pH}$ values no binding of the nitrophenols to DPPC was observed indicating that the interaction between PNP and DOMA is purely electrostatic in nature.

The average orientation of chromophores at the interface can be determined by measuring $\Delta R$ with plane polarized light under inclined incidence $[5,15]$. The spectra shown in Fig. 7 have been obtained for DOMA monolayers spread on subphases with a) ONP and b) PNP with the monofilm compressed to an initial surface pressure of $\Pi=20 \mathrm{mN} / \mathrm{m}$. The incident light is at an angle of $\alpha=45.1^{\circ}$ with respect to the normal. Curves 1 and 3 are for $p$-polarized light ( $E$ in the plane of incidence), curves 2 and 4 for $s$-polarized light ( $E$ perpendicular to the plane of incidence). Due to some instrumentation difficulties with regard to polarized light below $380 \mathrm{~nm}$ the spectra of PNP was recorded in the presence of $\beta$-cyclodextrin in the subphase. It is known that the PNP molecules go into the cavity of cyclodextrin molecules and the absorption maxima shifts to longer wavelength range [19]. PNP molecules may undergo a slight change in orientation when present in the cavity of the $\beta$-cyclodextrin. However, we found no change in the intensity of the reflection signal for light at normal incidence for PNP 


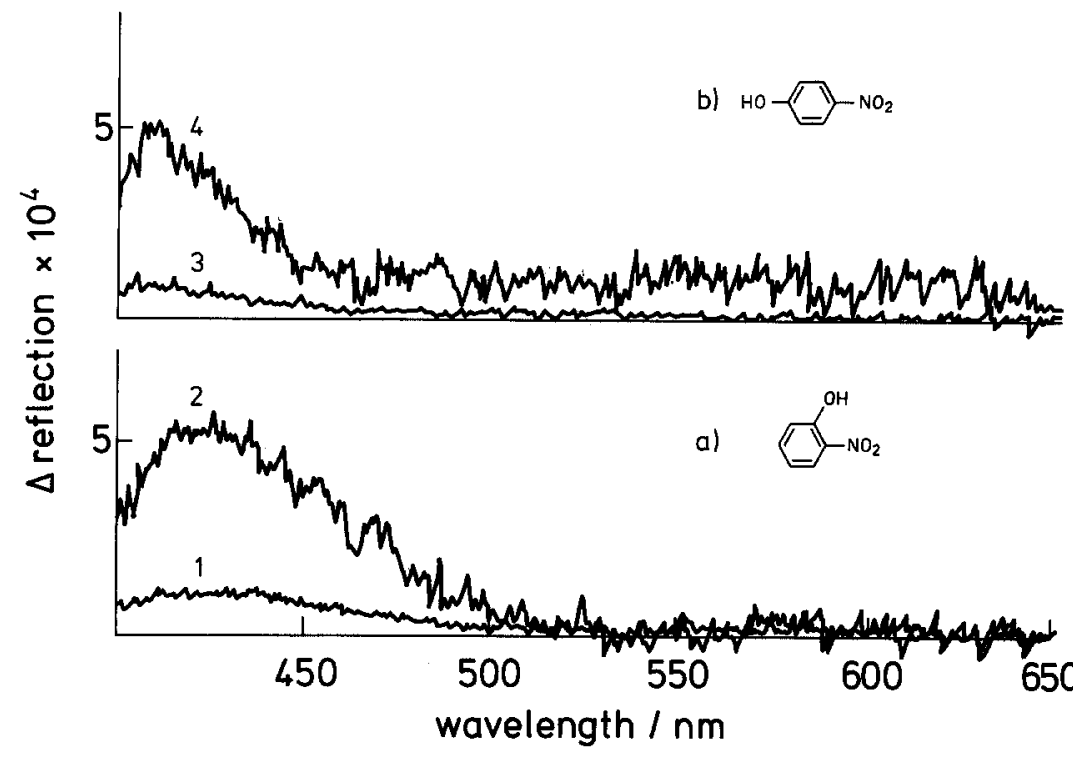

Fig. 7. Reflection spectra for linearly polarized light, angle of incidence $\alpha=45.1$ for nitro phenols (curves 1 and 3) $\Delta R_{p}$; (curves 2 and 4) $\Delta R_{s}$ at surface pressure $=20 \mathrm{mN} / \mathrm{m}$. Subphase a) ortho-nitrophenol $2 \times 10^{-4} \mathrm{M}$, and b) paranitrophenol, $2 \times 10^{-4} \mathrm{M}$ with $3 \times 10^{-3} \mathrm{M} \beta$ cyclodextrin, $\mathrm{pH}=10.0$ molecules adsorbed to the monolayers with or without cyclodextrin in the subphase. This could also mean that the PNP molecules are not completely included in the cavity. This seems to be in accordance with the recent $\mathrm{x}$-ray crystallographic work on nitrophenols complexing with cyclodextrins [20] according to which PNP molecules are not included in the cavity but are located in the intermolecular space between the host molecules. From Figs. 6 and 7 the ratio of $\Delta R_{s} / \Delta R_{p}$ for both PNP and ONP adsorbed to DOMA monolayers is estimated to be about 5 . According to the theoretical approach [5] the transition moments of the chromophores are oriented parallel to the layer plane when the ratio of $\Delta R_{s} / \Delta R_{p}$ is around 5 . Hence it can be said that the average orientation of the bound phenolate ions is parallel to the layer plane.

\section{Conclusion}

The reflection spectroscopy offers an elegant method for studying the adsorption behavior of such weakly surface active molecules like nitrophenols. The binding of ONP and PNP to positively charged monolayers indicates that electrostatic interactions are dominant. This binding takes place to both the fluid as well as the solid state of the monolayer. The transition moments of both bound isomers are oriented parallel to the substrate plane.

\section{Acknowledgement}

The support of this work by a fellowship of the Alexander von Humboldt Stiftung for one of us (Z.K.) is gratefully acknowledged. This work was partly funded by the Bundesministerium für Forschung und Technologie. (No. 03 8620).

\section{References}

1. Zeyer Y (1985) In: Bjorseth A, Angeletti G (eds) Organic micropollutants in the aquatic environment. D Reidel Publishing Company, Dordrecht, pp 305-311

2. Keith HL, Telliard WA (1979) Environ Sci Technol 13:416-423

3. Kuhn H, Möbius D, Bücher H (1972) In: Weissberger A, Rossiter B (eds) Physical methods of chemistry. Vol 1, part III B, Wiley, New York, pp 577-702

4. Grüniger H, Möbius D, Meyer H (1983)J Chem Phys 79:37013710

5. Orrit M, Möbius D, Lehmann U, MeyerH(1986)J Chem Phys 85:4966-4979

6. Fromherz P (1975) Rev Sci Instrum 46:1380-1385

7. Bhattacharya K, Sitzman EV, Eisenthal KB (1987) JChem Phys 87:1442-1443

8. Paluch M, Fielk M (1980) J Colloid Interface Sci 73:282-286

9. Marra J (1986) J Phys Chem $90: 2145-2150$

10. Harkins WD, Morgan J (1925) J Proc Natl Acad Sci11:637-642

11. Gaines Jr GL (1966) In: Insoluble monolayers at liquid-gas interface. Wiley-Interscience, New York

12. Davies JT, Rideal EK (1963) In: Interfacial phenomena. Academic press, New York

13. Möbius D, Grüniger $H$ (1984) Bioelectrochem Bioenerg 12:375-381

14. Möbius D, Orrit M, Grüniger H, Meyer H (1985) Thin Solid Films 132:41-53

15. Lehmann U (1988) Thin Solid Films 159:259-267 
16. Kozarac Z, Dhathathreyan A, Möbius D (1987) Eur Biophys J 15:193-196

17. Kozarac Z, Dhathathreyan A, Möbius D (1988) FEBS Lett 229:372-376

18. Kozarac Z, Dhathathreyan A, Möbius D (1988) Colloid and Surfaces 33:11-24

19. Cramer F, Saenger W, Spatz H (1967) J Am Chem Soc 89:14-50

20. Harata K (1988) Bull Chem Soc Japan 61:1939-1944
Received November 29, 1988; accepted January 16, 1989

Authors' address:

A. Dhathathreyan

Max-Planck-Institut, Biophysikalische Chemie

Postfach 2841

D-3400 Göttingen, F.R.G. 